\title{
An Expression Involving All Ordered Compositions of the First $n$ Ordered Natural Numbers and Two Classic Polynomials
}

\author{
Soumendra Bera \\ Mahishadal Raj College, Vidyasagar University, W.B., India
}

\begin{abstract}
Decomposition of $n !$ in terms of elementary symmetric polynomial of $n$ of $k$ integers: $1, \ldots, k$ helps to find an expression that involves with all ordered compositions of the first $n$ ordered natural numbers. The expression has a reduced version in terms of complete homogeneous symmetric polynomial of degree $n$ in 1, 2, $\ldots, k$; and thus there exists a relation between the polynomials of two kinds. In the context, we show an analogous pair of identities: one is a recurrence relation between Stirling numbers of both kinds and another one is a binomial coefficient identity.
\end{abstract}

Keywords: recurrence; sequence; ordered composition; factorial; symmetric polynomials, Stirling number of kind 1 and Stiring number of kind 2.

\section{Introduction}

We decompose $n$ ! in terms of elementary symmetric polynomial of $n$ of $k$ integers: $1, \ldots, k$. By the process of recursive substitution, the relation further helps to find an expression that involves with the ordered compositions of $n$ integers: $1,2, \ldots, n$ all. Further investigation for occurrences of the integers in the expression leads to reduce the coefficients in the forms of complete homogeneous symmetric polynomial of degree $n$ in 1 , $\ldots, k$; and to obtain a relation between the symmetric polynomials of two kinds. The relation is further useful to find a recurrence relation between Stirling numbers of two kinds. In the context, we show an analogous pair of identities at the end.

The first $n$ natural numbers are united in different ways in the paper. The readers can find: 'Diversity in Unity' in the paper.

\section{Expression Involving the Compositions of the First $n$ Ordered Natural Numbers: $1, \ldots, n$ from Decomposition of $\boldsymbol{n}$ ! for $\boldsymbol{n} \geq \mathbf{2}$}

For $n$ of $k$ variables: $x_{1}, \ldots, x_{k}$, elementary symmetric polynomial: $e_{n}\left(x_{1}, \ldots, x_{k}\right)$ or in brief $e_{n}\left\{x_{k}\right\}$ is defined formally by

$$
e_{n}\left\{x_{k}\right\}=\sum_{1 \leq i_{1}<i_{2}<\ldots<i_{n} \leq k} x_{i_{1}} x_{i_{2}} \ldots x_{i_{n}}
$$

Substituting $1, \ldots, k$ for $x_{1}, \ldots, x_{k}$, we get $e_{n}(1, \ldots, k)$ or in brief

$$
e_{n}\{k\}=\sum_{1 \leq i_{1}<i_{2}<\ldots<i_{n} \leq k} i_{1} i_{2} \ldots i_{n}
$$

Examples: $e_{1}\{4\}=1+2+3+4 ; \quad e_{2}\{4\}=1.2+1.3+1.4+2.3+2.4+3.4 ; e_{3}\{4\}=1.2 .3+1.2 .4+1.3 .4+$ 2.3.4; $e_{4}\{4\}=4$ !. The special values are: $e_{0}\{k\}=1$ and for $n>k, e_{n}\{k\}=0$.

We decompose $(n+1)$ ! in terms of the notation $e_{n}\{k\}$ and obtain the subsequent results as shown.

From (1), we get:

$$
\begin{aligned}
& (n+1) !=(1+n)(1+n-1) \ldots(1+2)(1+1) \\
& =1^{n}+e_{1}\{n\} 1^{n-1}+e_{2}\{n\} 1^{n-2}+\ldots+e_{n-1}\{n\} 1^{1}+e_{n}\{n\} \\
\Rightarrow & 1^{n}=(n+1) !-e_{1}\{n\} 1^{n-1}-\ldots-e_{n-1}\{n\} 1^{1}-e_{n}\{n\} .
\end{aligned}
$$

$$
\begin{aligned}
1 & =2 !-e_{1}\{1\} \\
1^{2} & =3 !-e_{1}\{2\} 1^{1}-e_{2}\{2\} . \\
1^{3} & =4 !-e_{1}\{3\} 1^{2}-e_{2}\{3\} 1-e_{3}\{3\} \\
1^{4} & =5 !-e_{1}\{4\} 1^{3}-e_{2}\{4\} 1^{2}-e_{3}\{4\} 1-e_{4}\{4\} .
\end{aligned}
$$

Now we plan to carry out the process of recursive substitution involving (1.1), (1.2), ... to obtain (2.2), (2.3), .. in succession. To maintain the order of the subsequent results, initially we re-write (1.1) by numbering it as (2.1): 


$$
1=2 !-e_{1}\{1\}
$$

Then the successive results are as shown.

(i) From (1.2) and (2.1), we get:

$$
\begin{aligned}
& 1^{3}=3 !-e_{1}\{2\}\left[2 !-e_{1}\{1\}\right]-e_{2}\{2\} \\
& \Rightarrow 1^{3}=3 !-2 ! e_{1}\{2\}+\left[e_{1}\{2\} e_{1}\{1\}-e_{2}\{2\}\right]
\end{aligned}
$$

(ii) In the $2^{\text {nd }}$ and $3^{\text {rd }}$ terms of (1.3), we replace $1^{2}$ and 1 by their expressions as in (2.2) and (2.1); and then

$$
\Rightarrow 1^{3}=4 !-3 ! e_{1}\{3\}+2 !\left[e_{1}\{3\} e_{1}\{2\}-e_{2}\{3\}\right]-\left[e_{1}\{3\} e_{1}\{2\} e_{1}\{1\}-e_{1}\{3\} e_{2}\{2\}-e_{2}\{3\} e_{1}\{1\}+e_{3}\{3\}\right]
$$

(iii) Similarly in the $2^{\text {nd }}, 3^{\text {rd }}$ and $4^{\text {th }}$ terms of (1.4), we replace $1^{3}, 1^{2}$ and 1 by their expressions as in (2.3), (2.2) and (2.1); and then get:

$$
\begin{aligned}
1^{4}= & 5 !-4 ! e_{1}\{4\}+3 !\left[e_{1}\{4\} e_{1}\{3\}-e_{2}\{4\}\right]-2 !\left[e_{1}\{4\} e_{1}\{3\} e_{1}\{2\}-e_{1}\{4\} e_{2}\{3\}-e_{2}\{4\} e_{1}\{2\}+e_{3}\{4\}\right] \\
& +\left[e_{1}\{4\} e_{1}\{3\} e_{1}\{2\} e_{1}\{1\}-e_{1}\{4\} e_{1}\{3\} e_{2}\{2\}-e_{1}\{4\} e_{2}\{3\} e_{1}\{1\}+e_{1}\{4\} e_{3}\{3\}-e_{2}\{4\} e_{1}\{2\} e_{1}\{1\}\right. \\
& \left.+e_{2}\{4\} e_{2}\{2\}+e_{3}\{4\} e_{1}\{1\}-e_{4}\{4\}\right] \\
\cdots & \cdots \\
1^{n} & =(n+1) !-n ! e_{1}\{n\}+(n-1) !\left[e_{1}\{n\} e_{1}\{n-1\}-e_{2}\{n\}\right]-\ldots
\end{aligned}
$$

(2.4) is an alternating signs expression of 5 terms with the descending order of factorial notations: 5!, 4 !, 3 !, 2 ! and 1 ! as the coefficients in its successive terms. The coefficient of 1 ! or the last term of (2.4) is again an expansion of 8 terms. 8 sets of bottom indices in 8 terms of this expansion are: $(1,1,1,1),(1,1,2),(1,2,1)$, $(1,3),(2,1,1),(2,2),(3,1), 4$ such that $1+1+1+1=1+1+2=1+2+1=1+3=2+1+1=2+$ $2=3+1=4$; and evidently involve with all $2^{3}$ or 8 compositions of 4 in a particular order. In like manner the sets of bottom indices in the $4^{\text {th }}, 3^{\text {rd }}$, and $2^{\text {nd }}$ terms of (2.4) involve with the ordered compositions of 3,2 and 1 respectively. We notice also similar involvements of (2.3), (2.2) and (2.1) with the ordered compositions. In general the particular decomposition of $(n+1)$ ! for $n \geq 1$ can yield an expression (2.n) of $n+1$ terms for unity such that $n$ terms except the first one of the expression involve with the compositions of the first $n$ natural numbers: $1,2, \ldots, n$ in succession where the compositions of each number occur following a definite order.

The number of terms of an expansion, which occurs as the coefficient of $r$ ! for $n \geq r \geq 1$ in (2.n), is equal to the composition of the integer: $n-r+1$. That is, the number of terms $=2^{n-r}$. In a definite order, all $2^{n-}$ ${ }^{r}$ compositions of the integer: $n-r+1$ involve with $2^{n-r}$ sets of bottom indices of $2^{n-r}$ terms of the expansion. The general form of the terms of the expansion is:

$$
e_{i_{1}}\{n\} e_{i_{2}}\left\{n-i_{1}\right\} e_{i_{3}}\left\{n-i_{1}-i_{2}\right\} \ldots e_{i_{m}}\left\{n-i_{1}-\ldots-i_{m-1}\right\}, \quad i_{1}+i_{2}+\ldots+i_{m}=n-r+1 .
$$

Example: Let $n=4$ and $r=2$. Then we have: $n-r+1=3$.

In (2.4), the number of terms of the expansion, which occurs as the coefficient of $2 !$, is $2^{4-2}$ or 4 . Four compositions of 3 are $1+1+1,1+2,2+1$ and 3 . The compositions are written in a definite order such that the successive four terms of the expansion are:

$$
\begin{gathered}
e_{1}\{4\} e_{1}\{4-1\} e_{1}\{4-1-1\}, e_{1}\{4\} e_{2}\{4-1\}, e_{2}\{4\} e_{1}\{4-2\}, \text { and } e_{3}\{4\} . \\
\text { Or, } \\
e_{1}\{4\} e_{1}\{3\} e_{1}\{2\}, \quad e_{1}\{4\} e_{2}\{3\}, \quad e_{2}\{4\} e_{1}\{2\}, \text { and } e_{3}\{4\} .
\end{gathered}
$$

(a) Counting of the notations of the type $e_{n}\{k\}$ in (2.n)

Step1. We know that the number of the compositions of $n$ into $k$ parts or summands is $\left(\begin{array}{l}n-1 \\ k-1\end{array}\right)$. Consequently the number of summands in the compositions of $n$ into $k$ summands is $k\left(\begin{array}{l}n-1 \\ k-1\end{array}\right)$.

Then the number of summands in all $2^{n-1} C(n)$

$$
\begin{gathered}
=\sum_{k=1}^{n} k\left(\begin{array}{l}
n-1 \\
k-1
\end{array}\right) \\
=(n+1) 2^{n-2}
\end{gathered}
$$


Step 2: The compositions of $n$ integers: $1,2, \ldots, n$ all occur in (2.n). The bottom index of a notation of the type $e_{n}\{k\}$ is a summand of a composition among the compositions of all $n$ integers. Then from (3), we get:

Number of the notations of the type $e_{n}\{k\}$ in (2.n)

$=$ Number of the summands of the compositions of all $n$ integers: $1,2, \ldots, n$

$$
=\sum_{n 2^{n-1}}^{n}(i+1) 2^{i-2}
$$

Remark 1: (4) implies a curious number- sum relationship:

Number of the summands of the compositions of all $n$ integers: $1,2, \ldots, n$

$=$ Sum of the summands of the compositions of $n$.

\section{Ordered Compositions}

The significant order of the compositions of $n$ or in brief $\operatorname{SOC}(n)$ is demonstrated in the paper: Soumendra era, "Relationships between Ordered Compositions and Fibonacci Numbers", Journal of Mathematics Research (JMR) under CCSE, Vol. 7, No.3, 2015. (2.1), (2.2), (2.3), ... involve with the compositions of the integers in a definite order. Rule for the significant order of compositions of a positive integer $n$ is stated below.

Rule for SOC(n): Under SOC(n), the summands of the $1^{\text {st }} C(n)$ are all 1; the last $C(n)$ is $n$ itself; and for $n \geq r \geq 2$, if any $k^{\text {th }} C(n)$ is: $x_{1}+\ldots+x_{r}$ then $(k+1)^{\text {th }} C(n)$ is: $x_{1}+\ldots+x_{r-2}$ $+\left(x_{r-1}+1\right)+$ the sum of $x_{r}-1$ summands which are all 1 such that if $r \geq 3$ then the first $r-$ 2 summands of $k^{\text {th }} C(n)$ appear also in $(k+1)^{\text {th }} C(n)$ in the same order, but if $r=2$ then such common summands of $k^{\text {th }} C(n)$ and $(k+1)^{\text {th }} C(n)$ cannot exist. The number of summands of $k^{\text {th }}$ $C(n)$ and $(k+1)^{\text {th }} C(n)$ under $S O C(n)$ are $r$ and $r+x_{r}-2$ respectively.

Example: We use the symbol of equivalence ( $\equiv)$ between $S O C(n)$ and its implication. We show an example below for $n=5$.

$\operatorname{SOC}(5) \equiv 1+1+1+1+1,1+1+1+2,1+1+2+1,1+1+3,1+2+1+1,1+2+2,1+3+1,1$ $+4,2+1+1+1,2+1+2,2+2+1,2+3,3+1+1,3+2,4+1,5$.

In Topic II, the sets of bottom indices in the successive two terms except the $1^{\text {st }}$ one of (2.2) involve with $S O C(1)$ and $S O C(2)$; in like manner three terms of (2.3) with $S O C(1), S O C(2)$ and $S O C(3)$; four terms of (2.4) with $S O C(1), \operatorname{SOC}(2), \operatorname{SOC}(3)$ and $S O C(4)$; and so on.

\section{Another Way of Analysis for Occurrences of the Integers in the Recurrence Expressions and a Reduced Version of (2.n)}

We have noticed that the terms in the expansions that occur as the coefficients of 1 !, 2 !, ... in (2.1), (2.2), (2.3),.. involve with the ordered compositions of $1,2,3, \ldots$; and with the ordered integers that have close connection with the summands of compositions. Here we investigate occurrences of the integers in the successive expressions in a different way.

Let us confine our attention to the coefficient of 2 ! in (2.4). We find that 1,2 and 3 occur as the bottom indices; and 2, 3 and 4 occur inside the braces. Although the numbers of occurrences of the distinct integers as the bottom indices and inside the braces \{\} are different yet the distinct integers are consecutive; and the number of distinct integers as the bottom indices is equal to the number of distinct integers inside the braces. So we use a symbolic abbreviation for the coefficient of 2 ! in (2.4) in the form:

$E$ [Set of distinct integers as the bottom indices, this inside the braces \{\}$]$.

Using the notation, which is indeed an arithmetic function, we may write a recurrence relation for the coefficient of 2 ! in the following form.

$$
E[(1,2,3),(2,3,4)]=e_{1}\{4\} E[(1,2),(2,3)]-e_{2}\{4\} E[(1),(2)]+e_{3}\{4\}
$$

In like manner the coefficient of 3 ! in (2.4) is:

$$
E[(1,2),(3,4)]=e_{1}\{4\} E[(1),(3)]-e_{2}\{4\} .
$$

The last term or coefficient of 1 ! in (2.4) is:

$$
E[(1,2,3,4),(1,2,3,4)]=e_{1}\{4\} E[(1,2,3),(1,2,3)]-e_{2}\{4\} E[(1,2),(1,2)]+e_{3}\{4\} E[(1),(1)]-e_{4}\{4\} .
$$


The coefficient of 4 ! in (2.4) is:

$$
E[(1),(4)]=e_{1}\{4\} .
$$

Similarly we can find the relations of the same kind for the coefficients of (i) 1 ! in (2.1), (ii) 1 ! and 2! in (2.2), and (iii) 1 !, 2! and 3 ! in (2.3). We further notice that inside the brackets: [ ] of the arithmetic functions, the consecutive integers of the $1^{\text {st }}$ set start with unity and the consecutive integers in the $2^{\text {nd }}$ set start with different integers. In other words we have:

$E$ [set of distinct integers as the bottom indices; this inside the braces]

$=E[(1,2, \ldots, n),(k, k+1, \ldots, k+n-1)]$, which contains two variables: $n$ and $k$.

Then we can write a reduced version: $F(n, k)$ for the arithmetic function such that

$$
F(n, k)=E[(1,2, \ldots, n),(k, k+1, \ldots, k+n-1)] .
$$

Using (6), we can immediately write the reduced forms of (5.1), (5.2), (5.3) and (5.4) in succession as shown.

$$
\begin{aligned}
& F(3,2)=e_{1}\{4\} F(2,2)-e_{2}\{4\} F(1,2)+e_{3}\{4\} . \\
& F(2,3)=e_{1}\{4\} F(1,3)-e_{2}\{4\} . \\
& F(4,1)=e_{1}\{4\} F(3,1)-e_{2}\{4\} F(2,1)+e_{3}\{4\} F(1,1)-e_{4}\{4\} \\
& F(1,4)=e_{1}\{4\}
\end{aligned}
$$

We obtain the above results for (2.4). We can also obtain the similar results for (2.3), (2.2) and (2.1). In general we obtain: for all $n, k \in \mathbb{N}$,

and

$$
F(1, k)=e_{1}\{k\} .
$$

$$
F(n+1, k)=\sum_{i=1}^{n}(-1)^{i-1} e_{i}\{n+k\} F(n+1-i, k)+(-1)^{n} e_{n+1}\{n+k\}
$$

We find that the coefficients of 4 !, 3!, 2! and1! in $(2.4)$ are $F(1,4), F(2,3), F(3,2)$ and $F(4,1)$ respectively. Similarly we can find that the coefficients of 3 !, 2 ! and1! in $(2.3)$ are $F(1,3), F(2,2)$ and $F(3,1)$; and these of 2 ! and 1 ! in $(2.2)$ are $F(1,2)$ and $F(2,1)$ respectively. Thus the reduced forms of $(2.1),(2.2),(2.3)$ and (2.4) are:

$$
\begin{aligned}
& 1=2 !-F(1,1) . \\
& 1^{2}=3 !-2 ! F(1,2)+F(2,1) . \\
& 1^{3}=4 !-3 ! F(1,3)+2 ! F(2,2)-F(3,1) . \\
& 1^{4}=5 !-4 ! F(1,4)+3 ! F(2,3)-2 ! F(3,2)+F(4,1) .
\end{aligned}
$$

We can verify the results by the process of recursive substitution. For instance, replacing $1^{2}$ and 1 from the $2^{\text {nd }}$ and $3^{\text {rd }}$ terms of (1.3) by their expressions as in (8.2) and (8.1) and then applying (7.1) and (7.2), we get (8.3). Thus the reduced versions of $(2.1),(2.2), \ldots$ are $(8.1),(8.2), \ldots$ in succession. The general form of $(8.1),(8.2), \ldots$ is:

Now we need to solve $F(n, k)$.

$$
1=(n+1) !+\sum_{j=1}^{n}(-1)^{j}(n+1-j) ! F(j, n+1-j)
$$

(a) Solution for $\boldsymbol{F}(\boldsymbol{n}, \boldsymbol{k})$

The solution of $F(n, k)$ is Theorem 3 which is the consequence of Theorem 1 and Theorem 2 .

Theorem 1: $F(n, 1)=1$.

Proof: We have: 
Putting $x=1$, we get;

$$
(x-1)(x-2) \ldots(x-n)=\sum_{i=o}^{n}(-1)^{i} e_{i}\{n\} x^{n-i} .
$$

$$
\sum_{i=1}^{n}(-1)^{i-1} e_{i}\{n\}=1
$$

(10) is useful to prove the theorem by induction. From (7.1) and (7.2), we have $F(1,1)=F(2,1)=1$. Hence the theorem is true for $n=1$ and for $n=2$. To complete the proof, we assume that the theorem holds for all $n \in \mathbb{N}$ with $1 \leq n \leq r$. Then we deduce that

The theorem follows. I

$$
\begin{aligned}
F(r+1,1) & =\sum_{i=1}^{r}(-1)^{i-1} e_{i}\{r+1\} F(r+1-i, 1)+(-1)^{r} e_{r+1}\{r+1\} \\
= & \sum_{i=1}^{r}(-1)^{i-1} e_{i}\{r+1\} \cdot 1+(-1)^{r} e_{r+1}\{r+1\} \\
= & \sum_{i=1}^{r+1}(-1)^{i-1} e_{i}\{r+1\} \\
= & 1 .
\end{aligned}
$$

Theorem 2: $F(n+1, k+1)=F(n+1, k)+(k+1) F(n, k+1)$.

Proof. To prove the theorem, first we derive the fundamental identity (11) below. We have defined the notation $e_{n}\left\{x_{k}\right\}$ in Topic II. By the definition, the following laws hold. The number of terms of $e_{n}\left\{x_{k}\right\}$ is $\left(\begin{array}{l}k \\ n\end{array}\right)$. A term of $e_{n}$ $+1\left\{x_{k}\right\}$ for $k \geq n+1$ is also a term of $e_{n+1}\left\{x_{k+1}\right\}$, which does not contain $x_{k+1}$ as a factor; and if $x_{k+1}$ is multiplied with a term of $e_{n}\left\{x_{k}\right\}$ then the product is a term of $e_{n+1}\left\{x_{k+1}\right\}$, which contains $x_{k+1}$ as a factor. This implies that $e_{n+1}\left\{x_{k}\right\}$ is the sum of $\left(\begin{array}{c}k \\ n+1\end{array}\right)$ terms of $e_{n+1}\left\{x_{k+1}\right\}$ where none of these terms has a factor $x_{k+1}$; and $x_{k+1} e_{n}\left\{x_{k}\right\}$ is the sum of remaining $\left(\begin{array}{l}k \\ n\end{array}\right)$ terms of $e_{n+1}\left\{x_{k+1}\right\}$ where $x_{k+1}$ is a common factor of these terms. We have then the following fundamental identity from the laws.

$$
e_{n+1}\left\{x_{k+1}\right\}=e_{n+1}\left\{x_{k}\right\}+x_{k+1} e_{n}\left\{x_{k}\right\} .
$$

Substituting $1, \ldots, k+1$ for $x_{1}, \ldots, x_{k+1}$, we get:

$$
\begin{aligned}
& e_{n+1}\{k+1\}=e_{n+1}\{k\}+(k+1) e_{n}\{k\} \\
& \Rightarrow e_{n}\{k\}=e_{n}\{k-1\}+k e_{n-1}\{k-1\} \\
& \quad\left[e_{0}\{k\}=1 \text { and for } n>k, e_{n}\{k\}=0\right]
\end{aligned}
$$

Using (11.1) we shall now prove the theorem by induction on $n$.

When $n=1$ and $k$ is a fixed positive integer, from (7.2) we deduce that:

$$
\begin{aligned}
& F(2, k+1)=e_{1}\{k+2\} F(1, k+1)-e_{2}\{k+2\} \\
& =\left[e_{1}\{k+1\}+k+2\right] F(1, k+1)-\left[e_{2}\{k+1\}+(k+2) e_{1}\{k+1\}\right] \\
& =e_{1}\{k+1\}[F(1, k)+k+1]+(k+2) e_{1}\{k+1\}-e_{2}\{k+1\}-(k+2) e_{1}\{k+1\} \\
& =e_{1}\{k+1\} F(1, k)-e_{2}\{k+1\}+(k+1) e_{1}\{k+1\} \\
& =F(2, k)+(k+1) F(1, k+1)
\end{aligned}
$$

Hence the theorem is true for $n=1$ and a fixed $k$. We assume that the theorem is true for all $n \in \mathbb{N}$ with $1 \leq n$ $\leq m$ and a fixed $k$. That is, by inductive assumption, we have: 


$$
\begin{aligned}
& F(m+1, k+1)=F(m+1, k)+(k+1) F(m, k+1), \\
& F(m, k+1)=F(m, k)+(k+1) F(m-1, k+1), \\
& \ldots, \ldots, \\
& F(2, k+1)=F(2, k)+(k+1) F(1, k+1) .
\end{aligned}
$$

Then we shall show that the theorem holds for $n=m+1$ and a fixed $k$.

We deduce that

$$
\begin{aligned}
F(m+2, k+1)= & e_{1}\{m+k+2\} F(m+1, k+1)-e_{2}\{m+k+2\} F(m, k+1)+\ldots \\
& +(-1)^{m} e_{m+1}\{m+k+2\} F(1, k+1)+(-1)^{m+1} e_{m+2}\{m+k+2\} . \\
= & {\left[e_{1}\{m+k+1\}+(m+k+2)\right] F(m+1, k+1) } \\
& -\left[e_{2}\{m+k+1\}+(m+k+2) e_{1}\{m+k+1\} F(m, k+1)+\ldots\right. \\
& +(-1)^{m}\left[e_{m+1}\{m+k+1\}+(m+k+2) e_{m}\{m+k+1\}\right] F(1, k+1) \\
& +(-1)^{m+1}\left[e_{m+2}\{m+k+1\}+(m+k+2) e_{m+1}\{m+k+1\}\right] . \\
= & e_{1}\{m+k+1\} F(m+1, k+1)-e_{2}\{m+k+1\} F(m, k+1)+\ldots \\
& +(-1)^{m} e_{m+1}\{m+k+1\} F(1, k+1)+(-1)^{m+1} e_{m+2}\{m+k+1\} \\
& +(m+k+2) F(m+1, k+1)-(m+k+2) F(m+1, k+1) \\
= & e_{1}\{m+k+1\}[F(m+1, k)+(k+1) F(m, k+1)] \\
- & e_{2}\{m+k+1\}[F(m, k)+(k+1) F(m-1, k+1)]+\ldots \\
+ & (-1)^{m} e_{m+1}\{m+k+1\}[F(1, k)+(k+1)]+(-1)^{m+1} e_{m+2}\{m+k+1\} . \\
= & F(m+2, k)+(k+1) F(m+1, k+1) .
\end{aligned}
$$

Thus the theorem holds for $n=m+1$ and is proved by induction for all $n \in \mathbb{N}$. $k$ can be given any positive integer-value to obtain the result. Hence we have the theorem for all $n, k \in \mathbb{N}$. I

Theorem 3: $F(n, k)$ is complete homogeneous symmetric polynomial of degree $n$ in $1,2, \ldots, k$

Proof: Complete homogeneous symmetric polynomial: $h_{n}\left(x_{1}, \ldots, x_{k}\right)$ or in brief $h_{n}\left\{x_{k}\right\}$ is the sum of all distinct monomials of degree $n$ in the variables: $x_{1}, \ldots, x_{k}$. Formally

$$
h_{n}\left\{x_{k}\right\}=\sum_{1 \leq i_{1} \leq \ldots \leq i_{n} \leq k} x_{i_{1}} x_{i_{2}} \ldots x_{i_{n}} .
$$

By substituting $1, \ldots, k$ for $x_{1}, \ldots, x_{k}$, we have $h_{n}(1, \ldots, k)$ or in brief

$$
h_{n}\{k\}=\sum_{1 \leq i_{1} \leq i_{2} \leq \ldots \leq i_{n} \leq k} i_{1} i_{2} \ldots i_{n} .
$$

Some special values of $h_{n}\{k\}$ are: $h_{0}\{k\}=1 ; h_{n}\{0\}=0, n \pm 0 ; h_{1}\{k\}=1+\ldots+k=e_{1}\{k\} ;$ and $h_{n}\{1\}=$ 1. Let $j$ be a definite integer in $(1, \ldots, k)$. According to the definition of $h_{n}\{k\}$, if $j$ is multiplied with the terms of $h_{n}\{j\}$ then the products are some terms of $h_{n+1}\{k\}$. We know that the number of terms of $h_{n}\{k\}$ is $\left(\begin{array}{c}k+n-1 \\ n\end{array}\right)$. Hence $j h_{n}\{j\}$ is the sum of $\left(\begin{array}{c}j+n-1 \\ n\end{array}\right)$ among $\left(\begin{array}{l}k+n \\ n+1\end{array}\right)$ terms of $h_{n+1}\{k\}$. Since $j \in(1, \ldots, k)$, we have:

$$
h_{n+1}\{k\}=\sum_{j=1}^{k} j h_{n}\{j\}
$$


From (12),

$$
\Rightarrow h_{n}\{k\}=\sum_{j=1}^{k} j h_{n-1}\{j\}
$$

From Theorem 2,

$$
F(1, k)=e_{1}\{k\}=h_{n}\{k\}=1+\ldots+k .
$$

By Theorem 1,

$$
\begin{gathered}
\sum_{i=1}^{k}\left[F(n+1, i+1)-F(n+1, i)=\sum_{i=1}^{k}(i+1) F(n, i+1)\right. \\
\Rightarrow F(n+1, k+1)-F(n+1,1)=\sum_{i=1}^{k}(i+1) F(n, i+1) .
\end{gathered}
$$

Then

$$
F(n+1, k+1)=\sum_{i=1}^{k+1} i F(n, i) .
$$

Similarly

$$
\begin{aligned}
& F(2, k+1)=(k+1) F(1, k+1)+k F(1, k)+\ldots+1 \cdot F(1,1) \\
& =(k+1) h_{1}\{k+1\}+k h_{1}\{k\}+\ldots+1 \cdot h_{1}\{1\} \\
& =h_{2}\{k+1\} .
\end{aligned}
$$

$$
\begin{aligned}
& F(3, k+1)=(k+1) F(2, k+1)+\ldots+1 \cdot F(2,1) \\
& =(k+1) h_{2}\{k+1\}+\ldots+1 \cdot h_{2}\{1\} \\
& =h_{3}\{k+1\} .
\end{aligned}
$$

... $\cdots$

In general for all $n, k \in \mathbb{N}$,

$$
F(n, k+1)=h_{n}\{k+1\}
$$

By Theorem1, for all $n, k \in \mathbb{N}$,

This completes the proof. I

$$
F(n, k)=h_{n}\{k\}
$$

\section{(b) Two identities}

From (9) and Theorem 3, we get an identity for $h_{n}\{k\}$ :

$$
1=\sum_{\mathrm{j}=0}^{\mathrm{n}}(-1)^{j}(n+1-j) ! h_{j}\{n+1-j\}, n \in \mathbb{N}, \quad n \geq 1 .
$$

Since $h_{0}\{1\}=1$, (14) holds for $n=0$ also. (14) is the reduced version of (2.n)

From (7.1), (7.2) and Theorem 3, we get a recurrence relation for $h_{n}\{k\}$ and $e_{n}\{k\}$ :

$$
0=\sum_{\mathrm{j}=0}^{\mathrm{n}}(-1)^{j} e_{j}\{n+k-1\} h_{n-j}\{k\}, \quad n \gtreqless k, \quad n, k \in \mathbb{N} .
$$

We can find the general form of (15) by substituting the variables: $x_{1}, x_{2}, x_{3,}, \ldots$ for $1,2,3, \ldots$ as shown.

$$
\begin{aligned}
0= & \sum_{\mathrm{j}=0}^{\mathrm{n}}(-1)^{j} e_{j}\left\{x_{n+k-1}\right\} h_{n-j}\left\{x_{k}\right\}, \quad n \gtreqless k, \quad n, k \in \mathbb{N} . \\
& \quad\left[e_{j}\left\{x_{n+k-1}\right\}=e_{j}\left(x_{1}, x_{2}, \ldots, x_{n+k-1}\right), \quad h_{n-j}\left\{x_{k}\right\}=h_{n-j}\left(x_{1}, x_{2}, \ldots, x_{k}\right)\right]
\end{aligned}
$$

Remark 2: In the description of complete homogeneous symmetric polynomial in Wikipedia: https://en.wikipedia.org/wiki/Complete_homogeneous_symmetric_polynomial, 
the relation between complete homogeneous and elementary symmetric polynomials is given as:

Or in brief',

$$
\sum_{i=0}^{m}(-1)^{I} e_{i}\left(X_{1}, \ldots, X_{n}\right) h_{m-i}\left(X_{1}, \ldots, X_{n}\right)=0 .
$$

$$
\sum_{i=0}^{m}(-1)^{I} e_{i}\left\{X_{n}\right\} h_{m-i}\left\{X_{n}\right\}=0 .
$$

Obviously (16) is different from (17)

\section{An Analogous Pair of Identities with the Same Integer Sequences and Different Mathematical Notations.}

One of the pair is a recurrence relation between Unsigned Stirling numbers of kind 1 and Stirling numbers of kind 2. The second one is a binomial coefficient identity. First we will show (i) the relation between $e_{n}\{k\}$ and the 'unsigned' or 'absolute value of' Stirling numbers of the first kind: $|s(n, k)|$ (with a small $s$ ); and (ii) the relation between $h_{n}\{k\}$ and Stirling numbers of the second kind: $S(n, k)$ (with a capital $S$ ). Then we will obtain the desired recurrence relation from (15).

\section{(i) Relation between $e_{n}\{k\}$ and $|s(n, k)|$ (with a small $s$ );}

Other notations for unsigned Stirling numbers of the first kind are: $\mathrm{c}(n, k)$ and $\left[\begin{array}{l}n \\ k\end{array}\right]$. We use here the notation: $\left[\begin{array}{l}n \\ k\end{array}\right]$ to show the relation.

Relation between signed and unsigned $1^{\text {st }}$ kind is:

$$
s(n, k)=(-1)^{n-k}\left[\begin{array}{l}
n \\
k
\end{array}\right] .
$$

The generating series for the unsigned $1^{\text {st }}$ kind is:

$$
(x)^{(n)}=\sum_{k=0}^{n-1}\left[\begin{array}{c}
n \\
k+1
\end{array}\right] x^{k+1} .
$$

$(x)^{(n)}$ is the notation of rising factorial.

$$
(x)^{(n)}=x(x+1)(x+2) \quad \ldots(x+n-1) .
$$

From the definition of $e_{k}\{n\}$,

$$
x(x+1)(x+2) \ldots(x+n-1)=\sum_{k=0}^{n-1} e_{n-k-1}\{n-1\} x^{k+1} .
$$

From (18.2), (18.3) and (18.4),

$$
\begin{gathered}
{\left[\begin{array}{c}
n \\
k+1
\end{array}\right]=e_{n-k-1}\{n-1\}, \quad n>k \geq 0} \\
\Rightarrow e_{k}\{n\}=\left[\begin{array}{c}
n+1 \\
n-k+1
\end{array}\right], \quad n>k \geq 0 .
\end{gathered}
$$

Remark 3: In the description of Stirling numbers of the first kind in Wikipedia: https://en.wikipedia.org/Stirling_numbers_of_the_first_kind ,

the relation between rising factorial. and unsigned Stirling number is given as:

$$
(x)^{(n)}=\sum_{k=0}^{n}\left[\begin{array}{l}
n \\
k
\end{array}\right] x^{k} .
$$

In fact, (18.2) is (19) with a minor variation by the inclusion of the extra term in (19): $\left[\begin{array}{l}n \\ 0\end{array}\right] x^{0}$ which is equal to 0 .

(ii) Relation between $e_{n}\{k\}$ and $S(n, k)$ (with a capital $S$ ).

Other notation for the Stirling numbers of the second kind is $\left\{\begin{array}{l}n \\ k\end{array}\right\}$. Here we use $\left\{\begin{array}{l}n \\ k\end{array}\right\}$. We recall the relation (7) in the paper: Soumendra Bera, 'Pairs of Comparable Relations for Complete Homogeneous 
Symmetric Polynomial', Journal of Mathematics Research (Canadian Center of Science and Education) Vol. 7 , No. 4, 2015, page 30:

$$
h_{k}\{n\}=\left\{\begin{array}{c}
n+k \\
n
\end{array}\right\}, \quad n \gtreqless k, \quad n, k \in \mathbb{N} .
$$

[The special values are: $h_{0}\{n\}=1$; and $h_{k}\{0\}=0, k \neq 0$.]

From(15), (18.5) and (20), we get the relation recurrence relation between unsigned Stirling numbers of kind 1 and Stirling numbers of kind 2:

$$
\begin{aligned}
0 & =\sum_{j=0}^{n}(-1)^{j}\left[\begin{array}{c}
n+k \\
n+k-j
\end{array}\right]\left\{\begin{array}{c}
n+k-j \\
k
\end{array}\right\} \\
\Rightarrow 0 & =\sum_{j=0}^{n-k}(-1)^{j}\left[\begin{array}{c}
n \\
n-j
\end{array}\right]\left\{\begin{array}{c}
n-j \\
k
\end{array}\right\}, \quad n>k \geq 0 .
\end{aligned}
$$

(21) has a mathematical beauty in its triangular representation involving the ordered integers. For example, when $n=4$ and $k \in(0,1,2,3)$ then the triangular picture of the relation is:

$$
\begin{gathered}
{\left[\begin{array}{l}
4 \\
4
\end{array}\right]\left\{\begin{array}{l}
4 \\
0
\end{array}\right\}-\left[\begin{array}{l}
4 \\
3
\end{array}\right]\left\{\begin{array}{l}
3 \\
0
\end{array}\right\}+\left[\begin{array}{l}
4 \\
2
\end{array}\right]\left\{\begin{array}{l}
2 \\
0
\end{array}\right\}-\left[\begin{array}{l}
4 \\
1
\end{array}\right]\left\{\begin{array}{l}
1 \\
0
\end{array}\right\}+\left[\begin{array}{l}
4 \\
0
\end{array}\right]\left\{\begin{array}{l}
0 \\
0
\end{array}\right\}} \\
=\left[\begin{array}{l}
4 \\
4
\end{array}\right]\left\{\begin{array}{l}
4 \\
1
\end{array}\right\}-\left[\begin{array}{l}
4 \\
3
\end{array}\right]\left\{\begin{array}{l}
3 \\
1
\end{array}\right\}+\left[\begin{array}{l}
4 \\
2
\end{array}\right]\left\{\begin{array}{l}
2 \\
1
\end{array}\right\}-\left[\begin{array}{l}
4 \\
1
\end{array}\right]\left\{\begin{array}{l}
1 \\
1
\end{array}\right\} \\
=\left[\begin{array}{l}
4 \\
4
\end{array}\right]\left\{\begin{array}{l}
4 \\
2
\end{array}\right\}-\left[\begin{array}{l}
4 \\
3
\end{array}\right]\left\{\begin{array}{l}
3 \\
2
\end{array}\right\}+\left[\begin{array}{l}
4 \\
2
\end{array}\right]\left\{\begin{array}{l}
2 \\
2
\end{array}\right\} \\
=\left[\begin{array}{l}
4 \\
4
\end{array}\right]\left\{\begin{array}{l}
4 \\
3
\end{array}\right\}-\left[\begin{array}{l}
4 \\
3
\end{array}\right]\left\{\begin{array}{l}
3 \\
3
\end{array}\right\} \\
=0
\end{gathered}
$$

Replacing all brackets: [ ] and braces: \{\} from the above relation by the parentheses of combinatorial notation: ( ), other relation with the same ordered integers in triangular frame is:

$$
\begin{gathered}
\left(\begin{array}{l}
4 \\
4
\end{array}\right)\left(\begin{array}{l}
4 \\
0
\end{array}\right)-\left(\begin{array}{l}
4 \\
3
\end{array}\right)\left(\begin{array}{l}
3 \\
0
\end{array}\right)+\left(\begin{array}{l}
4 \\
2
\end{array}\right)\left(\begin{array}{l}
2 \\
0
\end{array}\right)-\left(\begin{array}{l}
4 \\
1
\end{array}\right)\left(\begin{array}{l}
1 \\
0
\end{array}\right)+\left(\begin{array}{l}
4 \\
0
\end{array}\right)\left(\begin{array}{l}
0 \\
0
\end{array}\right) \\
=\left(\begin{array}{l}
4 \\
4
\end{array}\right)\left(\begin{array}{l}
4 \\
1
\end{array}\right)-\left(\begin{array}{l}
4 \\
3
\end{array}\right)\left(\begin{array}{l}
3 \\
1
\end{array}\right)+\left(\begin{array}{l}
4 \\
2
\end{array}\right)\left(\begin{array}{l}
2 \\
1
\end{array}\right)-\left(\begin{array}{l}
4 \\
1
\end{array}\right)\left(\begin{array}{l}
1 \\
1
\end{array}\right) \\
=\left(\begin{array}{l}
4 \\
4
\end{array}\right)\left(\begin{array}{l}
4 \\
2
\end{array}\right)-\left(\begin{array}{l}
4 \\
3
\end{array}\right)\left(\begin{array}{l}
3 \\
2
\end{array}\right)+\left(\begin{array}{l}
4 \\
2
\end{array}\right)\left(\begin{array}{l}
2 \\
2
\end{array}\right) \\
=\left(\begin{array}{l}
4 \\
4
\end{array}\right)\left(\begin{array}{l}
4 \\
3
\end{array}\right)-\left(\begin{array}{l}
4 \\
3
\end{array}\right)\left(\begin{array}{l}
3 \\
3
\end{array}\right) \\
=0
\end{gathered}
$$

$\left[\begin{array}{l}n \\ k\end{array}\right],\left\{\begin{array}{l}n \\ k\end{array}\right\}$ and $\left(\begin{array}{l}n \\ k\end{array}\right)$ imply different integers. Clearly if we substitute the integer-values for the notations: $\left[\begin{array}{l}n \\ k\end{array}\right],\left\{\begin{array}{l}n \\ k\end{array}\right\}$ and $\left(\begin{array}{l}n \\ k\end{array}\right)$, the initial sameness of the integer sequences of the above two triangular pictures will vanish; zero is yet the value of each expansion.

The second triangular picture is owing to the binomial coefficient identity:

$$
\sum_{j=0}^{n-k}(-1)^{j}\left(\begin{array}{c}
n \\
n-j
\end{array}\right)\left(\begin{array}{c}
n-j \\
k
\end{array}\right)=0, \quad n>k \geq 0 .
$$


(22) is the identity (1) with minor variation in the paper: Soumendra Bera, "A Typical Sequence of + and - signs, and an Application of the Powers of Twos in the Expression of a Positive Integer in Binary Scale", IOSR Journal of Mathematics (IOSR-JM) Volume 12, Issue 3 Ver. I (May. - Jun. 2016), page-3.

\section{References}

[1]. G.E. Andrews, The Theory of Partitions, Cambridge University Press, 1998, Chapter 4, p54

[2]. H.S.Hall and S.R. Knight, Higher Algebra, Macmillan and Co, London 1967, pp 164-165.

[3]. John F. Riordan. (1979). Combinatorial identities, R. E. Krieger Pub. Co

[4]. Khristo N. Boyadzhiev (2012). "Close encounters with the Stirling numbers of the second kind". Mathematics Magazine. 85 (4): pp.252-266.

[5]. M. Abramowitz, M. and I.A.Stegun (Eds.). "Stirling Numbers of the First kind and Second Kind." §24.1.3 and §24.1.4." in handbook of mathematical functions with formulas, graphs, mathemayicle Tables, $9^{\text {the }}$ printing New York: Dover, pp. 824-825, 1972.

[6]. Miklós Bóna, A walk through combinatorics, World Scientific Publishing Co. Pte. Ltd, 2011, PP 93-94

[7]. Ronald L. Graham, Donald E. Knuth, Oren Patashnik (1988) Concrete Mathematics, Addison-Wesley, Reading MA.ISBN 0-20114236-8, p. 244.

[8]. R. P. Stanley, Enumerative Combinatorics, Vol. 1. Cambridge, England: Cambridge University Press, 1997.

[9]. Serge Lang, Undergraduate Algebra, Third Edition, Springer 2005, p-159.

[10]. Sloane's A008275 and A008277: triangle read by rows of Stirling numbers of the first kind and second kind, The On-Line Encyclopedia of Integer Sequences. OEIS Foundation.

\section{Annexure}

1. Below is a triangular array of values for the Stirling numbers of the unsigned first kind (Sloane's sequence A008275 in the On-Line Encyclopedia of Integer Sequences) :

\begin{tabular}{|r|r|r|r|r|r|r|r|r|r|r|}
\hline $\mathbf{n} \backslash k$ & 0 & 1 & 2 & 3 & 4 & 5 & 6 & 7 & 8 & 9 \\
\hline $\mathbf{0}$ & 1 & & & & & & & & & \\
\hline $\mathbf{1}$ & 0 & 1 & & & & & & & & \\
\hline $\mathbf{2}$ & 0 & 1 & 1 & & & & & & & \\
\hline $\mathbf{3}$ & 0 & 2 & 3 & 1 & & & & & & \\
\hline $\mathbf{4}$ & 0 & 6 & 11 & 6 & 1 & & & & & \\
\hline $\mathbf{5}$ & 0 & 24 & 50 & 35 & 10 & 1 & & & & \\
\hline $\mathbf{6}$ & 0 & 120 & 274 & 225 & 85 & 15 & 1 & & & \\
\hline $\mathbf{7}$ & 0 & 720 & 1764 & 1624 & 735 & 175 & 21 & 1 & & \\
\hline $\mathbf{8}$ & 0 & 5040 & 13068 & 13132 & 6769 & 1960 & 322 & 28 & 1 & \\
\hline $\mathbf{9}$ & 0 & 40320 & 109584 & 118124 & 67284 & 22449 & 4536 & 546 & 36 & 1 \\
\hline
\end{tabular}

2. Below is a triangular array of values for the Stirling numbers of the second kind (Sloane's sequence A008277 in the OEIS ):

\begin{tabular}{|r|r|r|r|r|r|r|r|r|r|r|r|}
\hline $\mathbf{n} \backslash \mathrm{k}$ & 0 & 1 & 2 & 3 & 4 & 5 & 6 & 7 & 8 & 9 & 10 \\
\hline $\mathbf{0}$ & 1 & & & & & & & & & & \\
\hline $\mathbf{1}$ & 0 & 1 & & & & & & & & & \\
\hline $\mathbf{2}$ & 0 & 1 & 1 & & & & & & & & \\
\hline $\mathbf{3}$ & 0 & 1 & 3 & 1 & & & & & & & \\
\hline $\mathbf{4}$ & 0 & 1 & 7 & 6 & 1 & & & & & & \\
\hline $\mathbf{5}$ & 0 & 1 & 15 & 25 & 10 & 1 & & & & & \\
\hline $\mathbf{6}$ & 0 & 1 & 31 & 90 & 65 & 15 & 1 & & & & \\
\hline $\mathbf{7}$ & 0 & 1 & 63 & 301 & 350 & 140 & 21 & 1 & & & \\
\hline $\mathbf{8}$ & 0 & 1 & 127 & 966 & 1701 & 1050 & 266 & 28 & 1 & & \\
\hline $\mathbf{9}$ & 0 & 1 & 255 & 3025 & 7770 & 6951 & 2646 & 462 & 36 & 1 & \\
\hline
\end{tabular}

